\title{
CRUES ET INONDATIONS
}

\author{
A partir des XXIIIe Journées de l'hydraulique \\ de la Société Hydrotechnique de France \\ organisées à Nîmes
}

les 14, 15 et 16 septembre 1994

Sous le patronage de :

Monsieur le Préfet du Département du Gard

Monsieur le Président du Conseil Régional Languedoc-Roussillon

Monsieur le Président du Conseil Général du Gard

Monsieur le Président du Conseil d’administration de l'Agence de l’Eau Rhône-Méditerranée-Corse

Monsieur le Directeur de la Sécurité Civile au Ministère de I'Intérieur

Monsieur le Directeur de l'Eau au Ministère de l'Environnement

Monsieur le Directeur de la Prévention des Pollutions et des Risques au Ministère de l'Environnement

Monsieur le Président de la Chambre de Commerce et d'Industrie de Nîmes

Monsieur le Président du Directoire de la Compagnie Nationale d’aménagement du Bas Rhône-Languedoc (B.R.L.)

Monsieur le Directeur Général de la Compagnie Nationale du Rhône (C.N.R.)

Monsieur le Directeur Général de la Société d'Aménagement Urbain et Rural de la Région Méditerranée (SAUR)

Messieurs les Présidents des CICOR-EDF Languedoc-Roussillon et Provence-Alpes-Côte d'Azur 


\section{Crues et inondations}

Les XXIII Journées de l'hydraulique, congrès biennal de la Société hydrotechnique de France, avaient cette année pour thème "crues et inondations". Ce thème de réflexion était particulièrement opportun après un hiver 93-94 singulièrement "riche» en inondations. La ville de Nîmes, qui a connu un véritable cataclysme le 3 octobre 1988. avait été choisie comme lieu de rassemblement les 14, 15 et 16 septembre 1994. Les congressistes, plus de 300 personnes, ont d'ailleurs été accueillis par un orage et ont subi quelques violentes averses pendant la durée du congrès, comme une illustration des phénomènes méditerranéens. En dehors des séances de travail, deux sorties ont permis aux participants d'une part de visiter des digues de Camargue et d'autre part d'admirer le Rhône depuis le belvédère de la Caramude (Villeneuve-les-Avignon).

Le congrès a été également l'occasion de la remise des deux prix délivrés par la SHF. Le prix Henri MILON, destiné à distinguer une cuvre particulièrement intéressante, a été remis à $M$. Philippe Coussot pour ses travaux sur la rhéologie des boues et laves torrentielles et des écoulements diphasiques liquide-solide. Quant au grand prix d'hydrotechnique, destiné à reconnaître une activité riche et marquante en hydraulique, il a été attribué cette année à M. Raymond GRAS. A son actif on compte une série d'études qui ont commencé avec les ruptures de barrage pour se continuer dans l'environnement et l'écologie.

Le mercredi 14 septembre, le congrès s'est ouvert avec un exposé de M. FABRE, adjoint au maire de Nîmes et de P. SCHEURER, directeur du service Equipements structurants de la mairie de Nîmes, rappelant les événements du 3 octobre 1988 et montrant la pugnacité avec laquelle l'action a été menée pour surmonter les difficultés techniques et réglementaires ainsi que les problèmes de financement des plans de prévention élaborés. Cet exposé a montré que la ville est traversée par plusieurs cadereaux (cours d'eau intermittants) dont la couverture de certains était un obstacle à l'évacuation des eaux. Mais il n'est plus possible de les remettre à ciel ouvert. Les solutions retenues sont donc des retenues en amont des bassins versants, l'injection dans des avens existant au voisinage, l'utilisation de carrières, l'installation de grilles multiétages pour arrêter voitures, caddies... Il ressort de l'exposé que les solutions techniques existent mais que les projets achoppent sur le problème du financement. Les mairies ne peuvent en effet dégager tous les fonds nécessaires aux travaux.

Après cet exposé introductif, le congrès s'est organisé en quatre grandes questions qui représentent 102 communications rassemblées dans un recueil de 795 pages en deux volumes et qui ont donné lieu chacune à un rapport général :

— phénomènes hydrauliques

— conséquences positives et négatives

— remèdes actifs et passifs

- prévention et gestion.

\section{Phénomènes hydrauliques}

La première question s'est déroulée sous la présidence de M. MARBOUTY, ingénieur général de la météorologie et était précédée d'un exposé du rapporteur général, $M$. THIRRIOT, professeur à l'Institut national polytechnique de Toulouse. Ce dernier a mis en évidence les paramètres qui interviennent depuis les précipitations jusqu'à l'écoulement dans le lit de la rivière: influence de la pente, perméabilité du sol, coefficient de frottement, composition des sous-bassins...

La pluie comme genèse de débits de crue était ensuite au centre des interventions avec des exemples de crues récentes. Chaque intervenant était amené à exprimer en l'espace de cinq minutes l'essentiel de sa communication. Ces temps d'exposés très courts ont été la règle pour toutes les questions, ce qui nécessitait de dégager l'essentiel, mais n'a pas été sans entrâner quelques frustrations. Il était surtout question des crues dans les régions méditerranéennes (Italie, Cévennes, Vaison-la-Romaine, Ardèche, Corse) où les précipitations peuvent atteindre quelques centimètres en quelques heures, mais aussi dans quelques autres régions (Mississipi, Manche). Les vitesses atteintes par l'eau donnaient une idée de la puissance dévastatrice. Certaines communications ont mis en évidence la nécessité d'utiliser les moyens météorologiques disponibles (radar: images satellites) pour prévoir les crues et organiser la protection civile. C. COSANDEY, sur l'exemple d'un petit bassin versant cévenol, où la saturation se propage de l'amont vers l'aval et crée un seuil de pluie à partir duquel la crue monte très rapidement par ruissellement. Quant à M. Avias, il mettait l'accent sur l'importance de la géologie, l'existence d'une grande fracture dans les Cévennes favorisant les crues de l'Ardèche. Une crue sur cette rivière le 22 septembre 1992 a été quelque peu occultée par celle de Vaison-la-Romaine mais elle n'en était pas moins violente: elle s'est déroulée en cinq heures.

La crue de Vaison-la-Romaine de ce même 22 septembre 1992 était au centre de plusieurs communications en rai-

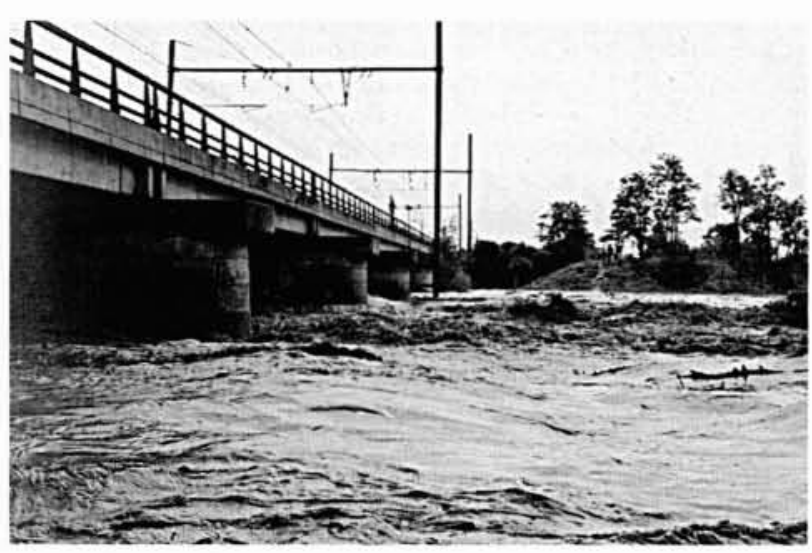

Le Roubion le $1^{\text {er }}$ octobre 1993 . Viaduc SNCF. 
son des dévastations engendrées. M. SENESI et col. ont insisté sur l'importance des aspects météorogologiques pour la prévision des crues. D'autres événements marquants dans les régions méditerranéennes ont été la pluie du 2.3 juin 1992 dans le région de Carcassonne où il est tombé $200 \mathrm{~mm}$ en cinq heures et les pluies et crues de la Toussaint 93 en Corsé. On a observé des phénomènes tels que le creusement d'un nouveau lit par un fleuve à travers in bois.

Les crues se produisant dans le nord de la France n'ont pas été pour autant oubliées avec l'intervention de P. GoubesviLie sur le bassin de la Douve (Manche). Les inondations y ont été fréquentes et leur contrôle doit tenir compte des portes à flot pour éviter l'introduction d'eaux marines dans les terres. Quant au Mississipi, il a connu en juillet 1993 une crue qui a fait beaucoup de victimes et des dégâts se montant à 12 milliards de dollars. Elle a été causée non par des pluies violentes mais par des pluies continuelles à raison de 200 à $500 \mathrm{~mm}$ par mois.

Le phénomène de ruissellement. en grande partie responsable des crues, a fait l'objet de quelques communications. Dans les terrains où la couche imperméable est à faible profondeur: il est intéressant de drainer les parcelles pour atténuer le ruissellement. Dans le bassin du Real Collobrier: on a observé les plus grands volumes d'écoulement à $20 \mathrm{~cm}$ de profondeur et non en surface et la formation de nappes perchées. L'énergie et la puissance dévastatrice des crues croît très vite avec la vitesse des

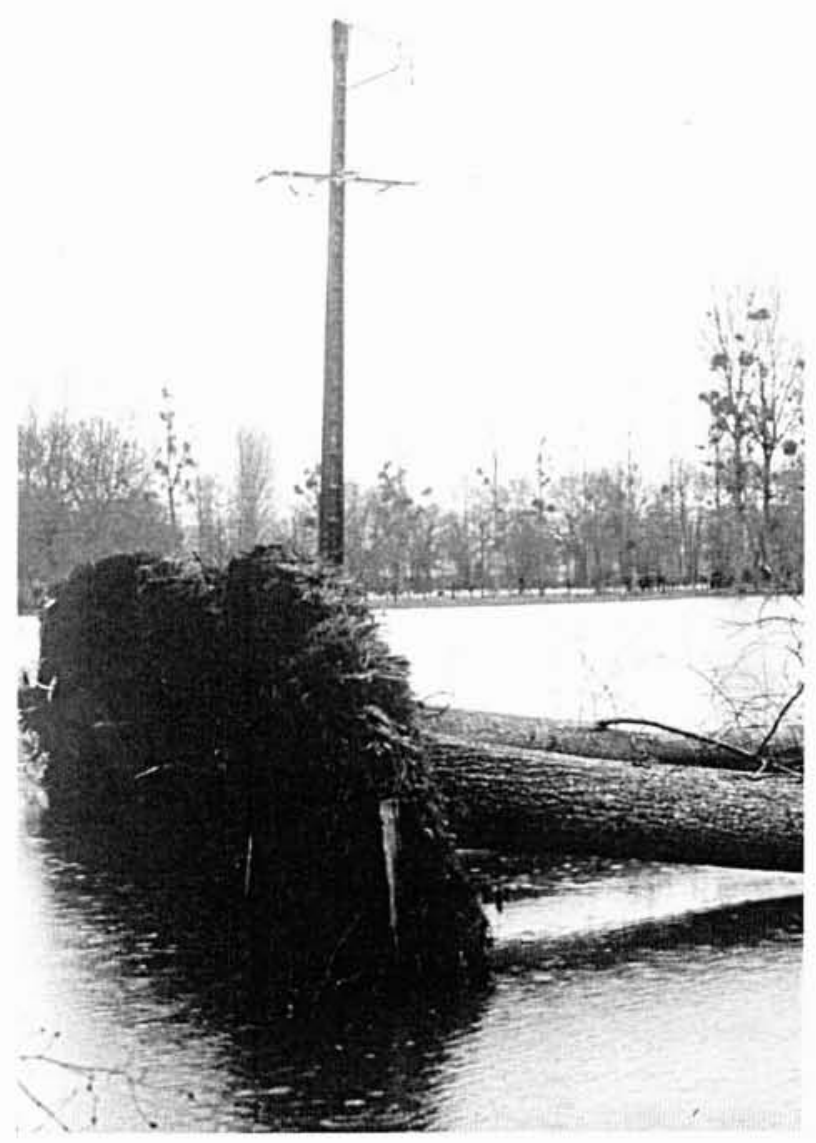

Arbre déraciné. Région de La Flèche (photothèque EDF - photo Marc Morceau). écoulements: si l'on dispose de renseignements sur les hauteurs d'eau. on manque cruellement d'informations sur les vitesses. Pour apprécier l'importance de ces vitesses. G. LALANNE conseille de mesurer la vitesse des corps flottants à partir de témoignages vidéo et des photographies aériennes en stéréo pour en déduire le débit. L'aprèsmidi, la projection d'une cassette vidéo sur la Bolivie a permis de mettre en évidence l'importance du transport solide qui fait monter le fond de la rivière quand celle-ci n'arrive pas à l'évacuer: On a observé également sur cette rivière la formation d'ondes très impressionnantes qualifiées de "roll waves" par certains.

Au-delà du phénomène physique des crues, le congrès traitait également de la modélisation des crues et des incertitudes statistiques et observations. F. CERNESSON proposait le modèle SHYPRE de relation pluie-débits dans lequel la pluie est assimilée à un phénomène aléatoire. D'autres intervenants présentaient l'un la modélisation de la réponse impulsionnelle d'un bassin versant. un autre une résolution implicite des équations de Saint-Venant pour la simulation des crues rapides, une troisième, des modèles aux éléments finis pour retrouver les débits mesurés, un autre, le modèle MERCEDES pour la résolution d'une partie des problèmes de non-linéarités spatiales et un cinquième, une simulation de rupture de barrages.

M. THIRRIOT a ensuite introduit le thème de la statistique en soulignant que dans la recherche de la fonction de répartition annuelle. il faut relativiser les événements qui ne semblent pas faire partie de la population (les horsains) et que l'on peut arriver à inclure avec certaines anamorphoses. Dans les interventions sur le sujet, les auteurs ont souligné l'importance de l'exploitation des données historiques et le risque que l'on prend en utilisant un modèle global de transformation pluie-débit en extrapolation. ainsi que l'intérêt d'appréhender des durées de retour d'un point de vue surfacique.

\section{Conséquences positives et négatives}

La question II concernant les conséquences positives et négatives, présidée par M. C. GLEIzEs, ingénieur général du génie rural. des eaux et des forêts et dont le rapporteur général était M. DECAMPS. Directeur du centre d'écologie des ressources renouvelables du CNRS de Toulouse, avait donné lieu à un plus faible nombre de communications (une douzaine). M. Décamps soulignait en effet dans son rapport que les crues posent d'énormes problèmes aux écologues qui peuvent les considérer soit comme des contraintes, soit comme des perturbations. selon l'échelle (bassin. réseau hydrographique, secteur de plaine inondable, habitat). Dans l'ensemble, les conséquences sont plutôt positives pour les écosystèmes. Les conséquences sur l'habitat n'ont donné lieu à aucune communication.

J.P. BRAVARD a d'abord souligné son inquiétude devant l'envahissement des plaines alluviales par la forêt en raison de la crise rurale et de l'abandon des activités au bord des rivières.

Les crues ont pour conséquence parfois la chenalisation de la rivière, comme par exemple sur la Garonne. Sur cette dernière. l'élargissement du chenal principal a entrâné la réduction de la surface inondable. Les aménagements hydrauliques ne sont pas non plus sans conséquences sur les paysages. Ainsi, une retenue à Artix sur le gave de Pau. 


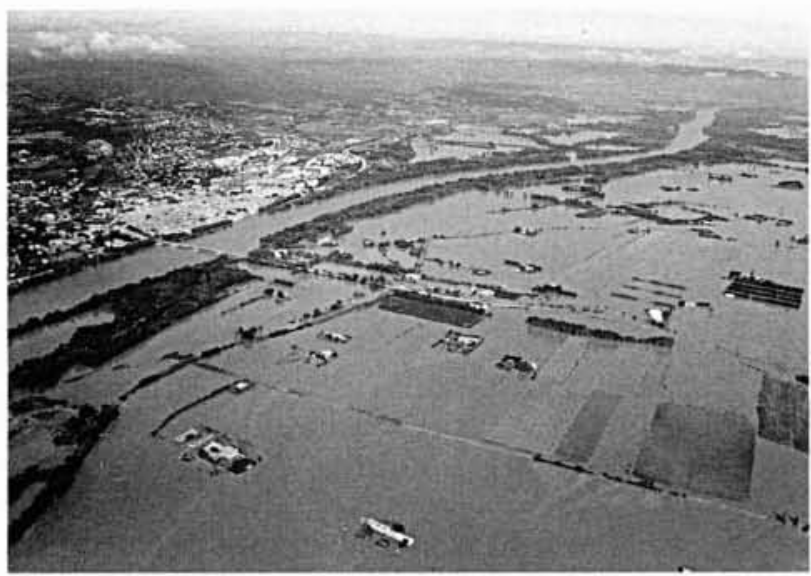

Inondation de la plaine de Pierrelatte (10 octobre 1993).

rivière à bras multiples enserrant une zone de saligue, a entrâné une progression de la sédimentation et des milieux boisés. On cherche à préserver l'avifaune qui s'était développée dans les premières années après la construction du barrage. La communication présentée par D. PEPIN a montré les conséquences d'un aménagement sur la Durance : envasement des retenues et surélévation des lits. En particulier l'étang de Berre connaît ce phénomène d'envasement suite à une déviation du cours de la Durance. Pour remédier à cet envasement, on a construit un bassin de décantation et on laisse dans la Durance toutes les eaux trop chargées. Sur le Drac, avant le confluent avec la Romanche, un barrage qui a supprimé les crues pendant des années a entraîné un développement de la végétation dans le lit en aval. Mais une crue en octobre 1993 a entraîné la dévastation des bois et les conséquences auraient pu être plus graves si la crue avait duré plus longtemps. Il faut donc entretenir les lits des fleuves.

Dans le domaine des conséquences nettement positives des crues, la communication de M. DAOUD a montré comment l'aménagement de dérivations du lit de l'oued El Fekka (Tunisie) permet de tirer le maximum de profit des crues en aidant l'eau à s'infiltrer et à recharger la nappe phréatique très sollicitée par l'agriculture.

Des études de cas ont suivi : Bordeaux (vignobles de l'agglomération), l'lle de France, Nîmes, Paris, Vaisonla-Romaine, le Grand Bornand, la Joséphina en Equateur. On a observé les murs de protection qui ont baissé ou se sont rehaussés selon les époques. Le cas de la Joséphina, vu à travers des diapositives, était le plus spectaculaire. Il s'agissait d'un éboulement de montagne ayant formé un barrage derrière lequel s'est formé un lac. Au bout d'un mois (on ne savait pas quel délai cela allait prendre), le barrage s'est rompu, provoquant une inondation catastrophique. Ces études de cas permettent de tirer des conclusions pour les cas voisins à venir.

\section{Remèdes actifs et passifs}

La question III abordait les remèdes. M. C. BocQuILlon, professeur à l'université de Montpellier, présidait la séance et M. E. TORMos, chef du secteur hydraulique de la CNR à Lyon, représentait $M$. LECORNU, ingénieur en chef des
Ponts et Chaussées. M. TORMOS a fait une brève introduction soulignant qu'un système de protection ne doit pas entraîner d'aggravation de la situation chez le voisin. Deux types de protection étaient envisagés : les remèdes de remodelage de terrain et les submersions organisées et contrôlées. Les choix économiques faisaient l'objet d'une troisième partie. On a regretté l'absence de nombreux intervenants qui auraient présenté des aménagements sur la Garonne, l'Isère, le Tarn, l'Ourthe et à la Réunion. Les autres intervenants ont insisté sur la nécessité d'entretenir le lit des rivières, de maintenir les endiguements, de mettre en place des plans de prévision et d'alerte de la population. Les déversoirs et les barrages régulateurs constituent des solutions complémentaires des endiguements. Mais la nécessité de voir les aménagements globalement sur un bassin est apparue car la protection d'un endroit précis peut entraîner une aggravation en amont ou en aval. M. PICARD a évoqué la rentabilité respective des digues et des barrages, faisant remarquer que les digues s'effondrent souvent. Dans le cas de la Vistre, qui coule près de Nîmes, un système d'étangs et de canaux qui s'étendent dans la zone de la petite Camargue jusqu'à la mer joue un rôle de laminage de crues. Le système est complexe à cause des fluctuations du niveau marin et la modélisation constitue un outil intéressant pour l'étude des protections en aval de Nîmes.

Les barrages construits pour la production d'électricité, tel celui de Serre-Ponçon dans les Alpes sur la Durance. constituent également un élément de protection contre les crues. Mais le rôle d'EDF n'est pas de maintenir ses barrages vides dans l'attente d'une forte pluie. C'est pourquoi les riverains ne comprennent pas toujours l'action d'EDF qui ouvre les vannes à l'approche d'une crue de façon à récupérer de la capacité de stockage. Pour être vraiment efficace dans la protection de Sisteron, le barrage de Serre-Ponçon devrait voir sa capacité augmentée. Sur le Rhône, les canaux de dérivation mis en place pour concentrer la hauteur d'eau et augmenter la possibilité de production d'électricité ne doivent pas aggraver la situation en cas de crue et si possible ralentir les effets. Une régulation a permis d'améliorer le temps de réaction.

Le remède des submersions contrôlées a été traité dans la partie suivante. Par exemple à Bordeaux, à la suite de plusieurs pluies exceptionnelles en 1982 puis 1986 des solutions ont été proposées pour éviter le ruissellement vers le centre ville ancien. Un bassin de stockage de $10000 \mathrm{~m}^{3}$ a été mis en place ainsi qu'un système à deux niveaux d'alerte. La submersion d'un passage souterrain est envisagée si nécessaire. Un autre exemple, dans le bassin de la Seine, montre comment une région d'exploitation agricole et de granulats, la Bassée, était utilisée comme zone d'expansion de crues. C'est une zone où l'on trouve des espèces rares d'animaux et qui comporte des gîtes aquiferes. Les agriculteurs se plaignent des crues mais il existe déjà deux barrages réservoirs en amont et il semble utile de laisser cette zone jouer son rôle d'expansion de crues et pourquoi pas d'en faire une zone de loisirs? Les barrages de retenue ont également été vus comme une solution pour protéger le système aval dans les cantons de Roquemaure et Villeneuve-les-Avignon.

Cependant, dans ce dernier cas comme dans les suivants, un plan d'occupation des sols bien pensé est fondamental pour éviter les catastrophes. Ainsi, les changements de pratiques agricoles avec des terrains cultivés s'étendant jusqu'au bord du Rhône ont été accusés par 


\section{CRUES ET INONDATIONS}

M. LEVASSEUR d'avoir provoqué une forte réaction du bassin du Rhône en 1994. En milieu urbain, les voiries surélevées pour être mises hors d'eau ont un effet pervers: elles diminuent les éléments de passage des eaux. De même, certaines constructions font obstacle à l'écoulement. M. DESBORDES suggère de prendre exemple sur les réalisations de la Grande-Bretagne et d'utiliser la voirie comme écoulement des eaux.

$L$ 'influence de la forêt de montagne sur la réduction des crues a visiblement intéressé l'assistance. La surface de forêt est en augmentation mais elle n'est utile pour la réduction des crues que lorsque la pente n'est pas trop forte. La forêt présente de plus l'avantage de produire du bois. Un inconvénient cependant : en cas d'incendie, la capacité d'absorption du sol est réduite et on observe la formation de laves torrentielles. M. COMBES ajoutait dans son intervention que les crues sont sensibles à tout processus de végétation et pas exclusivement à la forêt.

Un dernier remède évoqué à propos des cas d'un affluent de la Loire en amont d'Angers et du Sri Lanka sont les stations de pompage qui permettent de refouler les eaux dans un fleuve plus important.

La dernière partie de la question traitait plus spécifiquement des choix économiques à faire. La cartographie des zones inondables et le calcul économique des dommages entraînés par les crues ont été réalisés pour la région lle de France. Ils peuvent servir de base de réflexion. On a proposé d'autre part une méthode d'évaluation de la rentabilité économique d'un investissement de protection contre les crues.

\section{Prévention et gestion}

La dernière question, présidée par J. ESTIENNE, ingénieur général des ponts et chaussées et dont le rapporteur général était P.-A. ROCHE, directeur départemental de l'équipement de l'Aube, comportait de nombreuses communications sur la météorologie, la prévision de crues et la communication en période de crues. M. ROCHE $n$ 'a pas pu introduire longuement la question par manque de temps. Il a seulement rappelé que l'utilisation des radars pour la prévision des crues date de quarante ans, que la couverture par le réseau ARAMIS est insuffisante dans le sud-est de la France et que les prévisions constituent un moyen de limiter les dégâts.

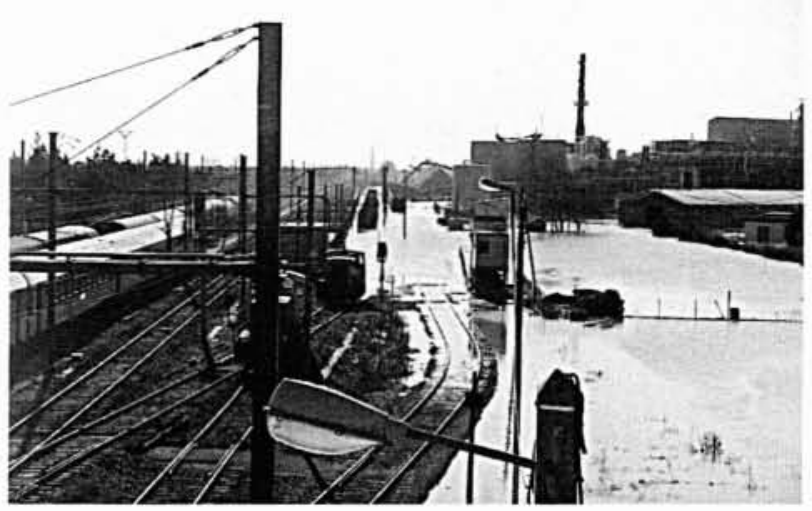

Tarascon le 8 janvier 1994. Usine de la cellulose.
La séance commençait par un film vidéo sur la prévision hydrométéorologique. Les communications suivantes présentaient l'utilisation du radar et ses problèmes (interception par l'isotherme $O^{\circ}$, problème de la bande brillante, échos de sol, de masque, mauvaises prévisions à Marseille à cause de l'éloignement du radar...). Des filtres permettent de mieux exploiter les images. Le logiciel CALAMAR offre la possibilité d'interpréter les informations en estimation de la lame d'eau. M. Andrieu a montré que les images radar donnent directement une image du stock d'eau et que dans le cas des Cévennes on peut prévoir à très court terme (une heure) la lame d'eau horaire.

Le service de prévision des crues s'est amélioré: les prévisions concernent à présent 18 bassins au lieu de quatre au départ. 53 services d'annonces de crues sont en place et la modernisation des réseaux se poursuit. Par exemple, dans le bassin de la Loire, on dénombre 120 stations équipées d'appareils de mesure et sept centres d'annonce de crues. Ces systèmes sont efficaces à condition que les appareils de mesure et les lignes de transmission ne soient pas en défaut (un cas a été cité de 15 postes totalement en défaut au même moment).

Plusieurs communications concernaient les modélisations pluie-débits (en continu ou par événement). Aucun des modèles présentés ne semble parfaitement satisfaisant. soit par une dynamique des crues un peu simplifiée, soit par une influence des conditions initiales mal prise en compte. M. CUNGE suggère d'utiliser divers types de mesure (au sol et images radars) et plusieurs modèles pour améliorer les prévisions. Certains intervenants ont déploré la rareté des prévisionnistes experts, l'informatique ne parvenant pas à remplacer l'expérience des hommes.

Les deux expériences les plus convaincantes concernent la ville de Bordeaux qui a mis en place un véritable outil de gestion de crise pour son réseau d'assainissement et le département de la Seine Saint-Denis qui exploite une méthode basée sur un système expert pour détecter les anomalies hydrauliques et pouvoir ajuster les télécommandes et téléréglages du réseau d'assainissement. Un autre exemple montrait que les systèmes de gestion de crise dépassent les frontières des pays développés. Ainsi, à Shangaï a été mis en place, avec l'aide de la Communauté européenne, un système pour gérer les risques d'inondations importants en raison de l'altitude basse de la ville et du risque de typhons. Le flux de données de mesure remonte au centre de prévision par un routeur de communication. Sont utilisés ensuite pour la prévision un modèle de transformation pluie-débit ainsi que des modèles chinois de systèmes typhons. En France, EDF peut servir d'interlocuteur en période de crise dans les zones où elle exploite une série de barrages. Le modèle LIDO développé par la Laboratoire national d'hydraulique est utilisé pour une modélisation hydraulique. Une vidange préventive du barrage peut être envisagée. Mais la gestion d'un ouvrage ne peut être efficace que si l'exploitant a des consignes claires.

La cartographie est également un outil fondamental puisqu'elle sert à identifier les zones à risque. Dans son introduction, P.-A. ROCHE a mis en avant le problème du morcellement des responsabilités communales alors qu'il serait souhaitable d'avoir des projets globaux de prévention prenant la dimension de l'amont et de l'aval. De plus, une formation de toute la population aux risques majeurs est à l'étude avec l'Education nationale. 


\section{CRUES ET INONDATIONS}

La cartographie sur ordinateur permet de visualiser en dynamique une crue, voir par exemple l'effet d'une rupture d'endiguement, l'inutilité d'une digue... La cartographie sert également à identifier les zones à risques en confrontant l'aléa et la vulnérabilité. Pour classer les zones à risque, on distingue les niveaux de risque acceptable et ceux qui sont acceptés pour les activités. Pour la vallée de la Loire, il existe un atlas des zones inondables. En SeineSaint-Denis, on a également une connaissance et un suivi des zones sensibles aux inondations. Pour sensibiliser les interlocuteurs, il faut en effet montrer des faits concrets, disposer d'informations quand ils sont demandeurs et pouvoir proposer des protections. Il faut aussi prévoir quelles sont les populations à déplacer pour les mettre en sécurité lors de crues exceptionnelles (les alerter par radio ou éventuellement par téléphone).

\section{Table ronde}

Le congrès se terminait par une table ronde animée par J. CHAPON en présence de nombreux élus venus rejoindre les congressistes, dont J. BOUSQUET, maire de Nîmes. M. CHAPON, dans son introduction, a posé le problème de savoir si une crue est un phénomène naturel et a mis l'accent sur le fait que toute action de protection a des conséquences négatives. D'autre part, que faut-il privilégier: la prévention ou l'intervention rapide? Il a également souligné qu'il est nécessaire de ne pas alerter inutilement pour rester crédible et que les citoyens oublient trop vite les catastrophes. M. Bourrelier, intervenant ensuite, a posé plusieurs problèmes : peut-on éviter les morts dans les crues rapides et les dégâts dans les crues de plaine? Jusqu'où vont les performances des systèmes d'alerte? Il a déploré le manque de lieu d'expertise centrale pour rassembler les connaissances en hydrométéorologie. Ce lieu pourrait-il être la SHF?

Les présidents de séances ont été ensuite invités à donner les points forts de chaque question. Puis les divers intervenants de la table ronde ont donné leurs points de vue avant que le débat avec la salle ne s'engage. M. LAURENT a insisté sur l'importance de la maîtrise de l'urbanisation en zone inondable et de l'entretien des cours d'eau (travail normalement fait par les riverains qui dans lequel devraient s'impliquer les collectivités locales et les agences de l'eau). M. ESTIENNE a souligné la place grandissante de l'informatique mais en souhaitant que l'approche géologique ne soit pas oubliée pour autant. $M$. MESCLOS a rappelé l'action d'EDF dans les zones équipées d'ouvrages pour la production d'énergie. M. ROUSSEAU s'est étonné qu'on ne dise plus qu'il existe des solutions aux grandes crues et a souligné que les variabilités de débits sont importantes pour le maintien de la qualité de l'eau. M. Wolf a rappelé qu'il n'y a pas que des crueséclairs mais aussi des crues lentes comme celles de la Seine qui causent également des dommages. M. RouX a montré le rôle des agences de l'eau : entretien des milieux aquatiques, actions de sensibilisation, de développement des connaissances. Se pose cependant un problème de

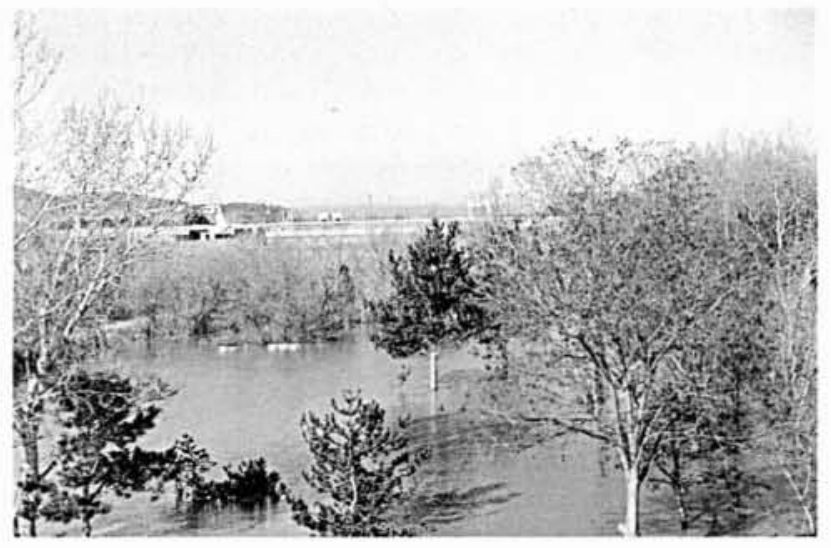

Tarascon le 8 janvier 1994. Usine de Vallabrègues. $11000 \mathrm{~m}^{3} / \mathrm{s}$.

financement des aménagements de protection car on ne peut pas faire payer indéfiniment le consommateur d'eau. Les bénéficiaires d'aménagements ou les responsables de mauvais aménagements pourraient alors être sollicités. $M$. TENIERE-DUCHAUX a ajouté que les compagnies d'assurance, qu'on avait déjà accusées au cours d'une session de ne pas financer la prévention, devraient aussi être sollicitées.

Des intervenants ont insisté sur l'importance de la sensibilisation des populations et de leur formation, sur la nécessaire valorisation de la profession des prévisionnistes. M. LEMPERIERE intervenant dans la salle a proposé trois mesures de prévention simples et peu cô̂teuses: afficher les hauteurs d'eau lors de précédentes crues dans les petites communes, désigner un responsable de la mairie pour se tenir au courant de la situation les jours à risque, mieux gérer les barrages existants et éventuellement en construire d'autres.

La table ronde a été clôturée par M. LAURENT, représentant le ministère de l'environnement, qui a donné les grandes orientations de la politique du ministère en matière de prévention des crues (décisions de janvier 1994): identification des risques, maîtrise de l'urbanisation, budget de cartographie doublé, contrôle par les préfets de l'urbanisation en zone inondée, décret de réglementation des campings en zone inondable, crédits exceptionnels pour la météorologie, l'alerte et l'instrumentation de massifs soumis à des glissements de terrain, aide exceptionnelle pour reprendre en main l'entretien des cours d'eau et moderniser les structures de gestion des ouvrages de protection. La possibilité d'exproprier pour cause de risques naturels majeurs fut aussi évoquée.

Il reste à souhaiter que la richesse des idées émises au cours de ce congrès ne sera pas perdue. Les lecteurs de ce numéro de la Houille Blanche qui n'ont pas assisté au congrès pourront compléter leur information dans les recueils de communications disponibles à la SHF et peut-être prendre contact avec certains intervenants.

Hélène Girardet 


\title{
$23^{\text {emes }}$ JOURNÉES DE L'HYDRAULIQUE «CRUES ET INONDATIONS »
}

\author{
PROGRAMME
}

\author{
QUESTION I \\ “PHÉNOMÈNES HYDRAULIQUES " \\ Président : D. MARBOUTY, Ingénieur général de la météorologie \\ Rapporteur général : C. THIRRIOT, Professeur à l'Institut National Polytechnique de Toulouse
}

A. - Pluies et genèse des débits de crue.

\section{1. - M.C. LLASAT et J. BARRANTES}

- Typologie des inondations dans le Nord-Est de l'Espagne : caractéristiques météorologiques

2. - M. DESBORDES et J.M. MASSON

- Fortes précipitations dans le Sud de la France.

3. - L. LANZA et F. SICCARDI

"Floods predictability in the Mediterranean area : the STORM'93 Euro. pean experience ,

\section{4. - J.V. AVIAS}

* Rôle des facteurs géologiques dans les caractéristiques des crues; leur rôle déterminant dans les crues records de certaines rivières cévenoles et. notamment, de la rivière ARDECHE, affluent rive droite du fleuve RHONE

5. - C. COSANDEY

" Genèse des crues dans les bassins élémentaires en région de movenne montagne granitique méditerranéenne.

6. - E. LORRE, M.P. ARLOT, A. DUTERTRE, J.P. GÉRARD et D. ZIMMER

- Influence du drainage sur le ruissellement hivernal à l'échelle de la parcelle,

7. - J.M. GRÉSILLON, A. TAHA et F. LE MEILLOUR

* Rôles respectifs de l'infiltration et du ruissellement de surface dans la formation des crues

8. - P. GOURBESVILLE

* Origines, conséquences et gestion des inondations dans une situation hydrologique complexe: le cas du bassin versant de la Douve (Manche) $\%$.

9. - G. LALANNE-BERDOUTICQ

* Aspects méthodologiques de la reconstitution des écoulements des grandes crues catastrophiques

B. - Observations et incertitudes statistiques.

\section{0. - M. LANG et I. DESUROSNE}

- Esquisse des risques de crues à l'échelle euro-méditerranéenne: les premiers résultats du programme FRIEND-AHMY exploitant les modèles AGREGÉ et TPG.

\section{1. - C. CANELLAS et C. MERLIER}

" Evaluation et cartographie de valeurs extrêmes de précipitations *.

12. - D. DUBAND

"Pour une meilleure prise en compte de l'information hydrométéorologique historique sur les crues importantes des bassins supérieurs de certaines rivières à risque

13. - O. CAYLA

Calcul probabiliste des crues de projet: SPEED \%.

14. - T. LEVIANDIER

"Intégration de la variabilité des conditions génératrices de crues dans lestimation des crues n.

15. - V.A. STANESCU et V. UNGUREANU

"Une méthode d'analyse de la décomposition d'une onde de crue".
C. - De l'Ouvèze au Mississippi : l'actualité des crues.

16. - J.C. FLAGEOLLET, P. de FRAIPONT, P. GOURBESVILLE, D. RISSER, N. THOLEY et J. TRAUTMANN

* Analyse d'une catastrophe: la crue de l'Ouvèze à Vaison-laRomaine -

17. - J.J. MESNIL, O. GILARD, O. CAZAILLET, B. COUVERT et J.M. DUJARDIN

"Bassin de l'Ouvèze : analyse de la crue du 22 septembre 1992 .

18. - J.M. DUJARDIN

* Bassin de l'Ardèche, la crue du 22 septembre 1992.

19. - S. SENESI, P. BOUGEAULT, J.L. CHËZE, P. COSENTINO et R.M. THÉPENIER

"Aspects météorologiques d'une inondation rapide: le cas de Vaisonla-Romaine

20. - C. BENECH et A. SAINT-UPÉRY

" La crue du 26 septembre 1992 dans les Pyrénées Orientales, la plus forte depuis l'Aiguat d'octobre $1940 \mathrm{n}$.

21. - P. GOMBERT et M. THOMAS

"L'événement pluviométrique exceptionnel du 23 juin 1992 dans la région de Carcassonne ».

22. - M.D. LOYE-PILOT et C. PASQUIER

"Les pluies et crues exceptionnelles de la Toussaint 1993 en Corse".

23. - L. COLLEVATI

* La crue de l'Indre des 21 et 22 décembre 1982 .

24. - J. ASTIER

"La crue du Mississippi de juillet 1993 \%.

D. - Modélisation des crues : mythe ou réalité ?

25. - A. DUMBRAUSKAS, A. POVILAITIS et P. PUNYS

"Modèles de ruissellement superficiel de petits bassins versants $\left(A<I \mathrm{~km}^{2}\right)$.

26. - F. CERNESSON et J. LAVABRE

"Modèle SHYPRE: simulation de crues grâce à la modélisation pluiedébit $=$.

27. - J.M. GRÉSILLON, C. OBLED, J.M. NEYRET-GIGOT et F. LE MEILLOUR

"Variabilité de la fonction de transfert d'un bassin-versant. Analyse des causes et essai d'interprétation $\%$.

28. - A. PAQUIER

"Simulation numérique des crues rapides ".

29. - J.M. HERVOUET et C. MOULIN

"Modèles mathématiques bidimensionnels aux éléments finis pour l'ana. lyse des crues et des inondations

30. - C. BOUVIER, G. FUENTES et R. DOMINGUEZ

"MERCEDES: un modèle hydrologique d'analyse et de prévision de crues en milieu hétérogène ».

31. - O. ZEVALLOS

* Expériences de modélisation physique et mathématique pour la prévision de rupture de barrages $\%$. 


\section{QUESTION II}

\section{"CONSÉQUENCES POSITIVES ET NÉGATIVES "}

Président : C. GLEIZES, Ingénieur général du Génie rural, des Eaux et des Forêts

Rapporteur général : H. DÉCAMPS, Directeur du Centre d’Ecologie des Ressources Renouvelables. CNRS. Toulouse

\section{A. - Ecosystèmes et paysages.}

\section{1. - H. PIÉGAY, J.P. BRAVARD et P. DUPONT}

Les ripisylves et les crues dans la France du sud-est: de l'histoire à la gestion contemporaine

\section{2. - J. STEIGER et F. GAZELLE}

Evolution contemporaine d'un paysage fluvial sous l'effet de la dynamique des crues et des impacts humains

3. - A. DAOUD et J. TRAUTMANN

"Les aménagements hydrauliques en milieu semi-aride : exemple de la plaine de Sidi-Bouzid (hautes steppes tunisiennes) „.

4. - P.C. CHAMARD et M.F. COURE

"Le contrôle de l'inondation des plaines du Delta du Niger: la réalité et les risques ".

\section{B. - Ecoulements et dommages.}

5. - P. BREIL et A. MALAFOSSE

* Essai de typologie hydroécologique de la variabilité des écoulement. en rivières $\%$.

6. - B. LAMBLIN, P. LEFORT, M. BROUAT et M. DEBOSSE " Le lac d'Artix les crues et transports solides du Gave de Pau. Naissance, vieillissement, possibilités de préservation d'un écosystème artificiel

\section{7. - J.Y. DELACOUX et D. PÉPIN}

* Conséquences et apports solides pour la gestion de l'aménagement de la Durance en période de crue $»$

8. - J.L. PEIRY et H. VIVIAN

* Dynamique des crues et réduction de la capacité du chenal consécutive à la construction d'un barrage hydroélectrique : l'exemple du Drac inférieur en amont de Grenoble

\section{C. - Etudes de cas}

\section{9. - P. BOURGOGNE et D. FRÉSIERS}

- Les crues d'un grand cru! Oui l'eut cru?

10. - Ph. DEBAR, P. DESGRANGES, L. GAZULL et N. JULIEN

" Une approche originale à l'échelle de la région lle de France : cartographie et calcul économique des dommages des crues historiques.. 11. - M. GUITON

"Inondations, destructions et protections: ruissellement et risque majeur. Cas d'application : Nimes et Vaison-la-Romaine ".

12. - E. CADIER. O. ZEVALLOS et A. VACCA

"Le glissement de terrain et les inondations catastrophiques de la Josefina

\section{QUESTION III}

« REMÈDES ACTIFS et PASSIFS "

Président : C. BOCQUILLON, Professeur à l'Université de Montpellier

Rapporteur général : J. LECORNU, Ingénieur en chef des Ponts et Chaussées,

assisté de E. TORMOS, Chef du secteur hydraulique à la C.N.R., Lyon

A. - Correction des crues et inondations par des remèdes de remodelage des terrains (barrages écrêteurs, endiguements et problèmes connexes, recalibrages).

\section{1. - B. CHOLIN}

"A pluies exceptionnelles, mesures exceptionnelles $\%$

2. - B. DARTAU, B. VORON, ZX FANG et G. ROCQUELAIN

"Les protections contre les inondations du Vidourle ",

3. - R. CORNE, J.L. LESTRUAUT et J. DUVOISIN

Protection de l'agglomération agenaise contre les crues de la Garonne

4. - R. NICOLLE, J. CAPOLINI et O. CAZAILLET

"Zones inondables et aménagement de l'Isère dans la vallée du Grésivaudan $\cdots$

5. - J. DUVOISIN

"LA RÉUNION: L'endiguement des grandes ravines sur leur cône de déjection $\%$.

6. - J. M. HIVER et G. BERTRAND

"Protections contre les inondations de l'Ourthe. Moyens d'études et remèdes $\%$.

7. - J. PICARD et R. THEPOT

* Stratégie de protection des vals de Loire contre les crues catastrophiques

8. - J.F. GEOFFRE et D. CORNUAILLE

* Schéma d'aménagement hydraulique du Vistre entre Nîmes et la mer „.

9. - C. SISSAKIAN, J.F. SALLES-LAFONT, B. GONDOUIN et

B. BUFFIN

"Etude d'aménagement coordonné de la Vallée de I'Ubaye ".

\section{B. - Submersions organisées et contrôlées.}

\section{0. - J. MASSON et F. COLLOMBAT}

"Rôle de la retenue de SERRE-PONCON et de l'aménagement de la Durance sur la protection contre les crues de la Durance ».

11. - R. DROBOT, O. CEACHIR et C. TOMA

"Gestion des ondes de crue dans un système couple : Réservoir permanent-Réservoir temporaire
12. - D. FRÉSIERS et P. BOURGOGNE

"Passage Galin : une inondation contrôlée ".

13. - M. BENEDETTI et B. POMMET

"Le cas des cantons de Roquemaure et Villeneuve-les-Avignon ".

14. - GREBAR, C. PRIGENT et Y. REUMAUX

"Quel avenir pour la Bassée? Un enjeu pour l'lle de France. Evacuation des eaux pluviales en rive droite du Rhône

15. - E. MORIN

"Sud Maroc: conception des ouvrages de dérivation des crues .

16. - F. COMBES, A. HURAND et M. MEUNIER

* La forêt de montagne: un remède aux crues .

17. - M. DESBORDES

"Principales causes d'aggravation du risque d'inondation par ruissellement pluvial en milieu urbanisé

18. - J.M. COMPAS et J. VAUCORET

* Une longue expérience dans la maîtrise et le contrôle des submersions dans la vallée du Rhône ».

19. - L. LEVASSEUR et Ph. GENTY

“ Les grandes crues d'octobre 1993 et de janvier 1994 sur le Bas-Rhône aménagé ».

C. - Choix économiques.

20. - P. FAUCHER et J.M. SOTTY

* Méthode d'évaluation de la rentabilité économique d'un investissemen pour la protection contre les crues $\gg$.

21. - G. FABRE, P. SCHEURER et J.M. LEQUEUE

«Le dossier hydraulique de Nimes après le 3 octobre 1988.

22. - J.P. TORTEROTOT et P.A. ROCHE

"L'estimation du cô̂t des dommages dus aux inondations fluviales et l'analyse des incertitudes.

23. - G. GOUDY et A. GAULHET

"Protection contre les crues à l'aide de stations de pompage de grand débit $"$ 


\section{QUESTION IV}

"PRÉVENTION ET GESTION"

Président : J. ESTIENNE, Inspecteur général des Ponts et Chaussées

Rapporteur général : P.A. ROCHE, Directeur départemental de l'Equipement de l'Aube, Troyes

A. - Suivi et prévision immédiate des précipiations

\section{1. - J.J. VIDAL, L. BOURREL, H. SAUVAGEOT et J.P.} DUPOUYET

- Un nouvel outil pour l'annonce des crues: la télédétection par radar.

2. - F. BRESSAND et A. KAPFER

"Apport des données radar de pluie pour la prévision et la gestion des crues rapides

3. - D. FAURE, H. ANDRIEU et J.D. CREUTIN

"Comparaison d'estimations radar el pluviométriques de pluies horaires sur de petits bassins versants cévenols

4. - H. ANDRIEU, V. THAUVIN et M.N. FRENCH

- Application d'un modèle de prévision de pluie à très courte échéance au contexte cévenol t.

5. - P. TOURASSE, R. GARCON, C. OBLED, V. AUBONNET et C. DUFOUR

" Nouveaux outils pour la prévision hydrométéorologique et le suivi des systèmes précipitants

6. - M. KERDONCUFF, A. MARIE et F. SEVAULT

*Valorisation de l'information des radars météorologiques .

7. - J.D. CREUTIN, H. ANDRIEU et W. KRA.JEWSKI

"Prévision à très courte échéance des intensités pluvieuses: utilisation des informations des radars météorologiques

8. - G. DELRIEU, R. VEYRIER, M. QUEAU et D. FAURE

- Caractérisation des précipitations méditerranéennes à de fines échelles spatio-temporelles $\%$.

B. - Prévision des crues.

9. - D. DURAND, J.L. NĖGRE, J. CUNGE et A. KAPFER

- Une méthodologie et un système intégré de prévision de crues-éclairs. pour les services d'annonces des crues..

10. - J.P. DUPOUYET, P. COHEN, C. FELIU, P. GLIZE et

J.J. VIDAL

"Projet * SEC \#. Système multi-Expert pour la prévision des Crues. Bassin de la Garonne

11. - C. OBLED, J.Y. RODRIGUEZ et G.M. SAULNIER

" Une approche opérationnelle pour la modélisation des bassins versants sujets aux crues éclair ”.

12. - A. PINHEIRO

"Prévision des crues en temps réel à l'aide d'un modèle distribué ".

13. - X. YANG, C. MICHEL et P.A. ROCHE

- Une méthode d'adaptation d'un modèle conceptuel pluie-débit pour la prévision des crues en temps réel ..

14. - L. MOULIN et D. REINBOLD

- Le réseau de collecte de données hvdrométéorologiques du bassin de la Loire : bilan de l'exploitation et enseignement sur la fiabilité

15. - C. LALLEMENT et P. TOURASSE

"Comportement et utilisation opérationnelle d'un réseau hydroclimatologique en période de crise

16. - J.L. ROY

"Annonce des crues. Avantages et inconvénients du système a en chaine ». Proposition pour un renforcement $\infty$.

17. - C. THIRRIOT et H. HABAIEB

"Comparaison de simulation de prévision manuelle de crue par des groupes d'opérateurs de nationalités et d'expériences différentes 18. - G. GODON et M. ODIER

"Lannonce des crues: une des priorités du Ministère de l'Environnement en matière d'innondations.
19. - A.P. BOUILLOT, B. VORON et M. MELKI

"Réseau d'observation hydrologique et hydraulique de la Ville de Nìmes ".

C. - Gestion des ouvrages en milieu urbain et des aménagements hydrauliques en temps de crues.

20. - D. FRÉSIERS et P. BOURGOGNE

"RAMSÉS: un télécontrôle de crise ".

21. - J.M. DELATTRE, G. AURIAUX et P. VOIGNIER

"Evolution de la gestion des risques hydrauliques sur un réseau d'assainissement. L'expérience de la Seine Saint-Denis .

22. - X. CAILMAIL et P. LE DELLIOU

"Elaboration des consignes de crues. Prise en compte des situation. exceptionnelles.

23. - I. BECCHI, E. CAPORALI, L. CASTELLANI et F. CASTELLI

- Le contrôle hydrologique des crues soudaines : le cas de Florence

24. - J.L. RAHUEL, J.L. NÈGRE et J.A. CUNGE

"SHANGHAI: Gestion prévisionnelle des inondations".

25. - H. DANG VAN MIEN, E. LAPERROUSAZ et A LIBAUX

* Exploitation automatique d'une chaîne d'aménagements en période de crue $»$.

D. - Prévention, communication et gestion de crise.

26. - J. de SAINT-SEINE

* La gestion du risque inondation : le cas du Paillon à Nice.

27. - O. BAROZET, O. GILARD, P. GIVONE et M. SOMMIER

"Le modèle de gestion hydraulique du bassin versant de la Bourbre ". 28. - H. DUMAY

“ CALYPSEAU: outil d'identification et de prévision du risque d'inondation par interprétation cartographique

29. - B. CHASTAN, O. GILARD, P. GIVONE et G. OBERLIN "Le modèle inondabilité, présent et avenir ".

30. - J.N. GAUTIER et L. GAZULI

*Inondabilité : de la recherche au bureau d'étude.

31. - P. BOURGOGNE et D. FRÉSIERS

"Assurances tous risques *.

32. - A. FAURE-SOULET, M. MASSON, J. LAVABRE, P. GIVONE et O. GILARD

"Identification et évaluation sommaire des risques d'inondations liées au ruissellement périurbain et aux crues torrentielles $"$

33. - C. COGEZ, I. PEREZ et F. CHAUMEAU

"Le risque d'inondation en ville lors de fortes pluies : le situer, le quantifier. le faire connaitre ".

34. - G. GARRY et J. GRASSIN

La prise en compte du risque d'inondation dans l'urbanisme et le droit des sols :

35. - M. THOMAS et P. GOMBERT

- Quelles mesures préventives pour les bassins versants ruraux à réponse rapide? :

36. - H.P. WILLI, A. PETRASCHECK et J.P. JORDAN

"Nouvelle orientation dans le domaine de la protection contre les crues en Suisse. Recommandations et applications pratiques

37. - J. NOYELLE

"L'évolution des stratégies de prévention contre les inondations "

38. - B. de VANSSAY

* Information, prévention et reconstruction * si vis pacem, para bellum "*

Les textes complets des communications sont disponibles à la Société Hydrotechnique de France, 199, rue de Grenelle, 75007 Paris, Tél. : (1) 47051337. Fax: (1) 45569746 


\section{ACTUALITÉS}

Voici bientôt six ans, Nîmes connaissait des inondations catastrophiques dues à un phénomène météorologique exceptionnel qui s'est acharné sur notre Ville pendant plusieurs heures le matin du 3 octobre 1988.

La mobilisation de tous a permis à notre Cité de dépasser cet événement dramatique et de retrouver rapidement une vie normale.

Ces inondations ont fait l'objet de multiples études, et de recherches en tous domaines.

Aujourd'hui, nous sommes honorés d'accueillir le Congrès de la Société Hydrotechnique de France lors des $23^{e}$ Journées de l'Hydraulique.

Je souhaite aux membres de la Société, ainsi qu'à l'ensemble des intervenants - tous de haut niveau - qui vous feront part de leurs recherches, la plus cordiale bienvenue dans notre Ville. Elus et Fonctionnaires évoqueront devant vous les mesures prises à Nîmes et dans sa région depuis 1988, que ce soit en matière de réseau d'alerte ou de plan de lutte contre les inondations.

Depuis octobre 1988 d'autres événements graves se sont produits, qu'ils s'agissent de Vaison-laRomaine ou de la Camargue qui nous sont toutes deux si proches.

L'importance des travaux que vous avez accomplis sur ces cas d'espèce permettra, je n'en doute pas, de mieux prévoir et de mieux maîtriser les crues et les inondations, sujets de votre colloque.

J'espère, également, que vos assises vous donneront le loisir de découvrir l'aspect culturel des questions hydrauliques dans notre région : l'aqueduc romain du Pont du Gard terminé par son castellum nîmois demeure le plus fameux des témoignages hydrauliques antiques!

Soyez les plus cordialement bienvenus dans une Ville où le patrimoine historique et les technologies les plus avancées savent dialoguer:

Jean BOUSQUET

Député Maire de Nîmes

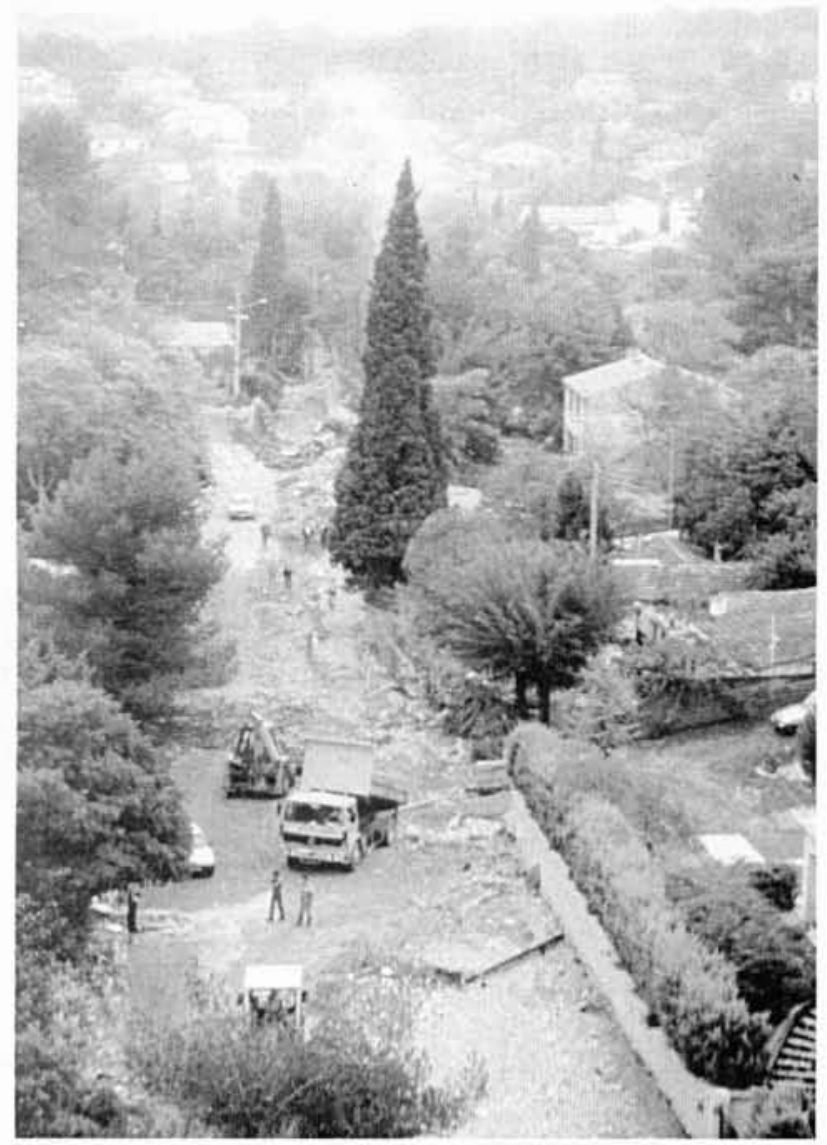

Nîmes après la catastrope d'octobre 1988 (photothèque EDF - photo Bertrand Clech). 


\title{
Allocution d'ouverture, des $23^{\mathrm{e}}$ Journées de l'hydraulique
}

\author{
Mesdames et Messieurs les Elus, Présidents et Directeurs, \\ Mesdames, Messieurs, Chers amis hydrauliciens,
}

L'objet de mon exposé est double.

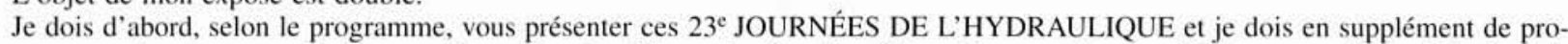
gramme - et pour ne pas surcharger d'exposés scientifiques la réception à laquelle vous êtes conviés tous ce soir par Monsieur le Maire de Nîmes - vous dire en quelques mots les mérites scientifiques des deux lauréats du Prix d'hydrologie et du Prix d'Hydrotechnique qui y seront couronnés.

Auparavant, je tiens à m'associer de tout cœur aux remerciements formulés par le Président Monsieur G. MAURIN, à tous ceux qui nous ont aidé à «monter » ces $23^{\text {e }}$ Journées de l'Hydraulique.

C'est la tradition de la Société Hydrotechnique de France d'appeler « Journées de l'Hydraulique " son congrès biennal tenu très régulièrement à ce rythme depuis 1950 . Si vous avez la curiosité de relever les titres de ces 23 Congrès, vous constaterez que tous font, pour les aspects techniques et scientifiques, référence à la Mécanique des Fluides et aux Techniques Industrielles qui y sont rattachées, mais que depuis 25 ans, 11 congrès sur 14 sont consacrés à des sujets d'environnement, si l'on entend ce terme au sens large des rapports entre l'eau, l'homme et la nature minérale et vivante.

Les $22^{\mathrm{c}}$ Journées, tenues dans l'amphithéâtre Henri Poincaré du Ministère de la Recherche, avec beaucoup de solennité en raison du $80^{\circ}$ Anniversaire de notre Société, dressaient un panorama à la fois exhaustif et prospectif de l'importance essentielle de l'eau dans la vie de notre planète, sous le titre très ambitieux de «L'Avenir de l'eau ».

Mais nous savons très bien que l'eau si nécessaire à la vie pourrait aussi en certaines circonstances engendrer des risques. L'un des membres de notre bureau, le Professeur THIRRIOT que vous verrez tout à l'heure à cette tribune, nous avait d'ailleurs proposé tout un programme sur «L'eau et ses risques ». De ce programme très général, nous n'avons retenu qu'un aspect: le risque lié aux excès de précipitations et d'écoulements, sous le titre très précis de "Crues et Inondations ».

Pourquoi ? Parce que ce sujet, qui fut l'objet d'études permanentes dans les Universités, les bureaux d'études publics et privés qui constituent une part importante des membres de la S.H.F., n'avait plus fait l'objet de manifestations publiques du type congrès depuis nos $10^{\mathrm{c}}$ Journées de l'Hydraulique tenues à Paris en 1968 sous le titre, d'ailleurs un peu plus restrictif qu'aujourd'hui, de "Prévisions des crues et protection contre les inondations ".

Depuis cette époque, de nombreuses évolutions avaient eu lieu non seulement dans les possibilités de traitements de données et de modélisation, mais aussi dans l'attitude des spécialistes à l'égard de ces phénomènes. On ressent nettement le passage d'un état d'esprit naguère fermement défensif à une volonté plus préventive. Ces changements - consacrés notamment par des modifications de structures qui, à partir de l'année 1984, confiaient l'ensemble des responsabilités et actions dans ce domaine au Ministère de l'Environnement - apparaissaient donc tout à fait utiles à reprendre.

A l'occasion d'ailleurs de ces modifications, la S.H.F. avait été chargée de réunir un groupe d'experts pour rédiger à l'attention des pouvoirs publics un GUIDE D'ANNONCE DES CRUES.

Peu après, des accidents très localisés mais très dramatiques, notamment en 1987 au Grand Bornand et en 1988 ici même à Nîmes, incitaient à une réflexion plus centrée sur les petits bassins et à une accélération des mesures curatives et surtout prédictives et préventives. La S.H.F. fut alors sollicitée par les Ministères de l'Environnement et de l'Intérieur pour organiser une nouvelle rencontre d'experts sur le thème «Crues et laves torrentielles " qui eut lieu en 1992.

C'est à cette époque, alors que nous commencions à tourner la page du précédent congrès, que nous décidions de rendre accessible au public tout ce travail de réflexion en l'enrichissant de ce que pourrait apporter un "appel à communications ". Le choix du lieu nous paraissait symbolique. Nous sommes très reconnaissants du bon accueil que la Ville de Nîmes a réservé à ce choix.

L'appel à communications était tout juste rédigé que surgissaient notamment les crues de l'Ouvèze avec les lourds dégâts spectaculaires que l'on sait, qui, bien entendu, ont fait l'objet de demandes très nombreuses de rapports.

Mais nous n'avions évidemment pas prévu que - le programme du congrès étant pratiquement clos — des crues exceptionnellement fortes se manifesteraient à l'automne 1993 en France continentale, en Corse, dans plusieurs pays d'Europe et simultanément aux Etats-Unis. Bien plus, tout près du lieu de notre congrès, le Rhône atteignait en janvier de cette année 1994, à Beaucaire, le débit de $11000 \mathrm{~m}^{3} / \mathrm{s}$ qui n'avait pas été observé depuis 1856 où il avait atteint $11640 \mathrm{~m}^{3} / \mathrm{s}$.

Ce calendrier montre bien que ce ne sont pas ces crues absolument récentes qui ont décidé de notre congrès et croyez bien que nous n'avons pas non plus assez d'influence pour les avoir provoquées, ni assez de science pour les avoir prédites.

Mais il était difficile d'être davantage inscrit dans l'actualité, actualité qui nous a d'ailleurs posé des problèmes : il nous a fallu en effet intégrer le mieux possible ces événements très récents par quelques communications de dernière minute et aussi par de courts voyages d'études (l'un en Camargue, l'autre en un point clé du Rhône à Villeneuve-lès-Avignon), que nous nous sommes efforcés, grâce au concours d'experts très compétents, de rendre très démonstratifs et très pédagogiques.

Qu'en soient remerciées toutes les personnalités qui ont accepté d'ajouter ces missions à leur lourde charge, notamment MM. Dambre, Pierron, Brassart et Agier pour la Camargue, MM. Savey, Gimenez et toute l'équipe de la C.N.R. pour le Rhône.

Je dois également remercier les rapporteurs généraux qui ont un rôle très lourd de préparation et d'orientation des discussions : MM. ThiRRIOT, DESCAMPS, LECORNu, TORMOS et Roche et les présidents de séance à qui nous devons toute l'ordonnance de nos débats : MM. Marbouty, Gleizes, BocQuillon et Estienne. Vous allez, dans très peu de temps, apprécier leurs grands talents d'animateurs et leur haute compétence scientifique.

Le sujet des inondations était tellement, ces dernières années, dans les esprits qu'il a certainement inspiré certains candidats aux Prix de la S.H.F. et peut-être sensibilisé les jurys au point que les deux lauréats que couronneront ce soir notre Président G. MAURIN et Monsieur le Maire de Nîmes le devront pour une part à leurs travaux sur les crues. 
La S.H.F. attribue régulièrement deux Prix d'Hydraulique : un Prix annuel d'hydrologie, bien connu des spécialistes sous le nom de Prix Henri MiLon, du nom de son fondateur Président de la S.H.F., en 1948, et un grand Prix d'Hydrotechnique remis traditionnellement à l'occasion de nos congrès à un ingénieur ou chercheur ayant apporté par l'ensemble de son œuvre une participation éminente aux progrès de l'hydraulique.

PRIX HENRI MILON

PRIX J'HYDROECHNIQUE

Le Prix annuel d'hydrologie est généralement remis à l'occasion d'un des trois colloques spécialisés que la S.H.F. organise chaque année. Mais l'ensemble des études qui motive l'attribution de ce Prix à Monsieur Philippe Coussơ est tellement inclus dans le thème de nos Journées qu'il est apparu opportun de faillir à nos traditions et de remettre ce Prix, hors programme, à la réception de ce soir.

En effet, le travail couronné concerne la « rhéologie des boues et laves torrentielles », domaine d'études extrêmement récentes et dont j'ai tout à l'heure souligné l'actualité. La thèse de rhéologie de M. Coussot permet, avec une explication des comportements, une étude très précise de tous les types d'écoulements polyphasiques, solides-liquides, depuis le totalement solide jusqu'à l'entièrement liquide. Partant d'une étude très complète des interactions au niveau des microstructures, puis d'une étude du comportement du fluide interstitiel des matériaux boueux, et d'une expérimentation sur le terrain permettant notamment la détermination du rôle des grosses particules, l'auteur arrive à une appréhension précise de la loi de comportement du complexe « fluides interstitiels plus blocs solides » tout à fait confirmée par de bons résultats d'expériences.

Cette thèse, complétée par une approche des lois d'écoulement des laves torrentielles puis par une étude précise des similitudes permettant la réalisation de modèles réduits, apporte un outil essentiel dans un domaine où le corps de connaissances scientifiques était encore très faible et permet de faire un progrès considérable là où les applications pratiques sont extrêmement utiles, comme nous le savons notamment par les accidents récents auxquels je faisais allusion tout à l'heure. Le travail de M. CoussoT avait déjà été remarqué dans les excellentes communications présentées par les équipes du CEMAGREF lors d'un récent colloque S.H.F. sur les « Transports solides » et c'est avec enthousiasme que le jury - auquel était présentée pourtant une dizaine de thèses de grande qualité - a tenu à attribuer le Prix Henri MiLon à ce travail exceptionnel.

Vous applaudirez ce soir Monsieur Philippe Coussot, comme vous applaudirez également avec la vigoureuse chaleur qui doit s'attacher à la plus haute distinction de la S.H.F., le lauréat du grand Prix d'Hydrotechnique Monsieur Raymond GRAS.

Conformément à son règlement, le grand Prix d'Hydrotechnique est attribué à un hydraulicien très confirmé pour l'ensemble de son œuvre afin de souligner l'apport d'un scientifique français de haut rang à la science hydraulique mondiale et les perspectives car ceux que nous couronnons sont encore en activité - que cet apport ouvre pour l'avenir.

Cette attribution se fait à la manière du Prix Nobel sans candidature, par une présélection basée sur la notoriété puis choix définitif par le jury.

Après Jean-Paul Huffenus, premier titulaire de ce Prix en 1982 - auquel je rends un hommage tout à fait particulier puisqu'après une vie en tous points exemplaire, il vient hélas de quitter cette terre - après Richard CANAVELIS, après l'équipe CHASSAING-Ha Minh puis Jean-Marie DelhaYe, Jacques LeCoffre, Pierre-Alain RoCHE et Alain VerRY, Raymond Gras a été retenu pour l'ensemble d'une cuvre particulièrement riche d'études de très haut niveau, marquée par la volonté de permettre la conception d'équipements industriels respectant et protégeant l'environnement.

Si j'ai dit tout à l'heure que son œuvre avait un lien avec le sujet de notre congrès c'est que sa thèse de doctorat complétée par deux communications à l'Académie des sciences portait sur l'étude des écoulements consécutifs à la rupture d'un barrage avec étude de l'influence des singularités de sections : un type de crue très particulier dont on parlera peu sans doute ici mais qui a donné lieu en France à tout un ensemble d'équipements et de mesures de prévention dont la conception repose pour l'essentiel sur le travail de Monsieur Gras.

Après cette œuvre initiale, et au cours d'une longue carrière à la Direction des Etudes et Recherches d'Electricité de France, Monsieur Gras a dominé, avec des responsabilités toujours croissantes et une audience internationale incontestée, tous les autres sujets liés au couple « usage de l'eau - environnement » et en particulier tous les problèmes de dispersion de chaleur dans les réfrigérants atmosphériques, dans les bassins de dissipation, dans le couplage "plans d'eau - atmosphère », dans les cours d'eau avec réalisation de modèles complets pour les plus grands fleuves et estuaires français et étrangers.

Passant de la physique à la biologie, il a étudié les mécanismes d'eutrophisation des cours d'eau et des lacs, l'influence du facteur thermique sur la photosynthèse et participé dans des équipes pluridisciplinaires avec le C.N.R.S., I'I.N.R.A., le CEMAGREF, à des recherches sur le comportement des écosystèmes aquatiques et des populations piscicoles aboutissant à des modélisations dynamiques et des méthodologies d'études globales de grands sites industriels intégrant qualité de l'eau, qualité de l'air, écologie terrestre et aquatique, et développement socio-économique.

Pour cette œuvre très complète, Monsieur GraS a été reconnu comme réalisant au mieux la synthèse des trois divisions de la S.H.F. : Mécanique des fluides, Applications industrielles, Ressources en eau, Aménagement et Environnement, et nous sommes particulièrement heureux de pouvoir souligner en lui la réunion, chère à la S.H.F., de l'eau fluide énergétique et de l'eau fluide de vie.

Monsieur Raymond GRAS, avec mes vives félicitations, je vous donne rendez-vous ce soir à l'hôtel de ville.

LANCE-

MENT DU

Maintenant, amis hydrauliciens, je vais vous laisser travailler. Vous savez qu'à la fin de vos débats, vendredi à partir de $10 \mathrm{~h}$, des personnalités éminentes informées par le canal des présidents de séance et des rapporteurs généraux des traits les plus marquants de vos discussions, débattront en Table Ronde pour préparer la clôture par le représentant de Monsieur le Ministre de l'Environnement.

Ce sera un temps très fort de ce congrès et $\mathrm{j}$ 'invite tous les spécialistes ici présents, rapporteurs ou participants, à rester à cette Table Ronde pour entourer de leur grande compétence technique les nombreuses personnalités du monde politique qui ont accepté notre invitation à cette clôture. Car l'essentiel de ce congrès, c'est vous participants venus en grand nombre de toutes les régions françaises, mais aussi de Suisse, de Belgique, de Chine, du Québec, d'Allemagne, du Maroc, du Mexique, de I'Equateur... (je présente mes excuses aux pays que j'aurais oubliés), c'est vous tous qui en avez assuré le contenu et qui allez en assurer le succès.

Je vous en remercie et je vous souhaite un fructueux travail dans l'assiduité sérieuse qui caractérise les hydrauliciens ; et aussi dans une ambiance de confrontation amicale et constructive à l'occasion des moments de studieuse détente qui vous sont offerts au cours de ces Journées.

Je vous souhaite un bon congrès. Merci. technique de la S.H.F. 